\begin{tabular}{|c|l|}
\hline Title & $\begin{array}{l}\text { Study on Nonlinear Electrical Characteristics of GaA s Based Three Branch Nanowire Junctions Controlled by Schottky } \\
\text { Wrap Gates }\end{array}$ \\
\hline Author(s) & Kasai, Seiya; Nakamura, Tatsuy a; Rahmani, Shaharin Fadzli Bin A bd; Shiratori, Yuta \\
\hline Citation & $\begin{array}{l}\text { Japanese Journal of A pplied Physics, 47(6), 4958-4964 } \\
\text { https://doi.org/10.1143/JAP.47.4958 }\end{array}$ \\
\hline Issue Date & 2008-06-25 \\
\hline Doc URL & http://hdl.handle.net/2115/38670 \\
\hline Rights & ○ 2008 The Japan Society of A pplied Physics \\
\hline Type & article (author version) \\
\hline File Information & JJA Pmm07080all.pdf \\
\hline
\end{tabular}

Instructions for use 


\title{
Study on Nonlinear Electrical Characteristics of GaAs-Based Three-Branch Nanowire Junctions Controlled by Schottky Wrap Gates
}

Seiya Kasai ${ }^{1,2}{ }^{*}$, Tatsuya Nakamura ${ }^{1}$, Shaharin Fadzli Bin Abd Rahman ${ }^{1}$, and Yuta Shiratori ${ }^{1}$

${ }^{1}$ Graduate School of Information Science and Technology and Research Center for Integrated Quantum Electronics (RCIQE), Hokkaido University,

North 14, West 9, Sapporo 060-0814, Japan

${ }^{2}$ PREST, Japan Science and Technology Agency (JST),

4-1-8 Honcho Kawaguchi, Saitama 332-0012, Japan

*E-mail address: kasai@rciqe.hokudai.ac.jp

\begin{abstract}
The nonlinear electrical characteristics of GaAs-based three-branch nanowire junction (TBJ) devices having Schottky wrap gates (WPGs) are investigated experimentally and theoretically, focusing on the nonlinear mechanism at room temperature in devices with large dimensions and the improvement of voltage transfer efficiency. Input-output voltage transfer curve, $V_{\text {out }}-V_{\text {in }}$, is characterized by changing nanowire width, $W$, temperature, $T$, and WPG gate voltage, $V_{\mathrm{G}}$, systematically. At room temperature, a bell-shaped $V_{\text {out }}-V_{\text {in }}$ voltage curve is observed even in the device having a nanowire width of $1,500 \mathrm{~nm}$, which is ten times larger than the electron mean free path. With decreasing wire width or temperature, the output curves are sharpened and curvature in the low-input-voltage region increases. The curvature rapidly increases and voltage transfer efficiency, $\Delta V_{\text {out }} / \Delta V_{\text {in }}$, approaches unity when $V_{\mathrm{G}}$ is decreased into the subthreshold region. A simple and compact model for the nonlinear characteristics in the nonballistic regime is introduced. The rapid change of the curvature and
\end{abstract}


complex curve in the subthreshold region under $V_{\mathrm{G}}$ control is due to the switching of the branch condition from resistive to capacitive by depletion underneath the WPG.

KEYWORDS: three-branch nanowire junction (TBJ), GaAs, Schottky wrap gate (WPG), nonlinear voltage transfer, analytical model 


\section{Introduction}

Three-branch nanowire junctions (TBJs) have been found to have unique nonlinear electrical characteristics recently, ${ }^{1,2}$ and they have attracted much attention as an important building block of nanowire-based devices and their integrated circuits. Experimentally clear nonlinear voltage transfer characteristics have been observed in TBJs made of III-V compound semiconductors ${ }^{3,4}$ and carbon nanotubes. ${ }^{5}$ The capability of high-speed operation was also demonstrated. ${ }^{4,6}$ On the basis of their functionality, their applications to various $\operatorname{digital}^{7,8}$ and analog circuits ${ }^{9,10}$ have been intensively investigated. At this stage, key issues include the understanding of the mechanism of the nonlinear characteristics and the improvement of voltage transfer efficiency. In order to control the nonlinear characteristics, we investigated Schottky wrap gate (WPG) control of a GaAs-based TBJ and succeeded in the electrostatic modulation of voltage transfer characteristics. ${ }^{11}$ On the other hand, contrary to the prediction ${ }^{1}$, the fabricated device having large dimensions compared with the electron mean free path exhibited clear nonlinear curves even at room temperature (RT). Such behaviors have been also observed in TBJ devices even without WPGs, and several investigations have been carried out on the mechanism, including the extension of the effective mean free path ${ }^{12}$ and velocity drop in the high-field domain. ${ }^{13}$ However, it has not been clarified yet. On the other hand, for their circuit application, a suitable device model applicable to circuit design is strongly required.

In this study, the nonlinear voltage transfer characteristics of GaAs-based TBJ devices having WPGs are investigated experimentally and theoretically, focusing on the nonlinear mechanism for devices with large dimensions at RT and the improvement of voltage transfer efficiency, $\Delta V_{\text {out }} / \Delta V_{\text {in. }} . V_{\text {out }}-V_{\text {in }}$ curves are characterized by changing nanowire width, $W$, temperature, $T$, and WPG gate voltage, $V_{\mathrm{G}}$. A mechanism for the nonlinearity of the present 
device is proposed and analysis is conducted on the experimental data on the basis of this model.

\section{Device Structure and Experimental Procedure}

Figure 1(a) shows an illustration of the TBJ device investigated in this study. It has a Tshaped three-branch nanowire junction, formed on an $\mathrm{AlGaAs} / \mathrm{GaAs}$ heterosturcture by etching. In order to control the device characteristics, nanometer-scale Schottky WPGs as shown in Fig. 1(b) are formed on right and left nanowire branches. The role of the WPG is to control the resistance by squeezing the nanowire branches electrostatically ${ }^{14}$ and it is expected to modulate the voltage transfer characteristics. The measurement circuit is also shown in Fig. 1(a). The TBJ device is operated by applying input voltage, $V_{\text {in }}$, to right and left branches in push-pull fashion and voltage in the center branch, $V_{\text {out }}$, is measured as output. According to the previous theory ${ }^{1}$, when the nanowire width is smaller than the electron mean free path and ballistic transport takes place in the junction, the output voltage is expected to show a bellshaped curve remaining always negative, such as $V_{\text {out }}=-\alpha V_{\text {in }}{ }^{2} / 2$, where $\alpha$ is the curvature. $\alpha$ is approximately given by $e / \mu$, where $\mu$ is the electrical chemical potential. ${ }^{1}$ This nonlinear voltage transfer characteristic comes from the difference of conductances for electrons entering and going out the center branch, because of the difference in the numbers of occupied quantum channels for left and right branches. On the basis of this theory, such nonlinear characteristic should disappear when the mean free path is shorter than the device size in increased temperatures.

For experimental study, T-shaped nanowire-branch junctions were formed on an $\mathrm{AlGaAs} / \mathrm{GaAs}$ heterostructure wafer by electron beam (EB) lithography and wet chemical etching. Geometrical nanowire width was varied from 90 to 2,000 nm. Nanowire length of 
left and right branches was typically $3 \mu \mathrm{m} . \mathrm{Cr} / \mathrm{Au}$ Schottky WPGs of $400 \mathrm{~nm}$ gate lengths with $800 \mathrm{~nm}$ spacing were formed on the left and right branches. The sheet carrier density of the unprocessed wafer at RT was $7.8 \times 10^{11} \mathrm{~cm}^{-2}$. The measured mobilities were $7,100 \mathrm{~cm}^{2} / \mathrm{Vs}$ at room temperature and $110,000 \mathrm{~cm}^{2} / \mathrm{Vs}$ at $77 \mathrm{~K}$, which corresponded to mean free paths of 80 and 1,500 nm, respectively. A scanning electron microscopy (SEM) image of the fabricated device is shown in Fig. 1(c). $V_{\text {out }}-V_{\text {in }}$ curves were characterized by varying geometrical nanowire width, $W$, temperature, $T$, and WPG voltage, $V_{\mathrm{G}}$.

\section{Results}

\subsection{Nanowire width dependence}

Figure 2(a) shows the $V_{\text {out }}-V_{\text {in }}$ curve for devices with various nanowire widths at RT. In this measurement, $V_{\mathrm{G}}$ was fixed at $0 \mathrm{~V}$. Fabricated devices exhibited clear bell-shaped curves even with the nanowire width of $1,500 \mathrm{~nm}$. In the devices whose $W$ was larger than the mean free path at RT, voltage transfer curves still changed depending on $W$. Voltage transfer curves became sharper systematically with decreasing $W$. Only the device with $W=2,000 \mathrm{~nm}$ showed an irregular curve. It should be noted that the output in the high-input-voltage region changed almost linearly against $V_{\text {in }}$ in devices with $W \leq 1,500 \mathrm{~nm}$. Derivatives of $V_{\text {out }}-V_{\text {in }}$ curves are plotted in Fig. 2(b). They clearly showed derivatives of unity in the high input voltage region. The input voltage range where the derivative was constant was widened by decreasing $W$. The observed nanowire width dependence was difficult to understand on the basis of the previous theory. Derivatives around $V_{\text {in }}=0 \mathrm{~V}$ indicated that the voltage transfer characteristics had higher-order components of $V_{\text {in }}$ than the parabolic function.

To discuss the wire width dependence quantitatively, measured $V_{\text {out }}-V_{\text {in }}$ curves were fitted by a parabolic function and curvature, $\alpha$, was evaluated. The result is plotted in Fig. 3 . The curvature systematically increased with decreasing $W$. It was almost proportional to $1 / W$. 
From this result, a small wire width is found to be favorable for logic applications because a voltage transfer efficiency of unity can be obtained even in the low-input-voltage region. However, there has been no analytical model explaining observed voltage transfer curves as well as such size dependence.

\subsection{Temperature dependence}

Figure 4 shows the $V_{\text {out }}-V_{\text {in }}$ curves of two different devices measured at various temperatures. Here, $V_{\mathrm{G}}$ was fixed at $0 \mathrm{~V}$. From the previous theory, the critical change of the voltage transfer characteristics was expected when the mean free path became longer than the nanowire width with the decrease in temperature. However, in the device with $W=570 \mathrm{~nm}$, the curves changed gradually depending on temperature and no critical change was observed. Curvature in the low-input-voltage region increased with decreasing temperature. The configuration of the curves at low temperatures was quite similar to those of narrow wire devices at RT in Fig. 2(a). This indicates that the wire width and temperature seem to have a similar effect on the nonlinear characteristics. The slope in the high-input-voltage region also became constant with decreasing temperature. On the other hand, the device with $W=120 \mathrm{~nm}$ showed a very weak temperature dependence. The nanowire width of this device was compatible to the mean free path even at RT. As temperature decreased from 300 to $200 \mathrm{~K}$, a small change was observed. When $T<200 \mathrm{~K}$, the curves were almost unchanged.

\subsection{WPG gate voltage dependence}

Figure 5(a) shows the measured $V_{\mathrm{G}}$ dependence of the $V_{\text {out }}-V_{\text {in }}$ curves of the fabricated device with $W=570 \mathrm{~nm}$ at RT. In this device, two WPG-controlled nanowire branches were confirmed to operate as conventional field-effect transistors (FETs) and the characteristics were similar to each other. Applying gate voltage to the right and left WPGs simultaneously 
and decreasing it, the curves were sharpened slightly when $V_{\mathrm{G}}>-0.6 \mathrm{~V}$. On the other hand, the device exhibited complex behavior when $V_{\mathrm{G}}<-0.6 \mathrm{~V}$. In this case, the observed curves seemed to contain two curves with different curvatures. Voltage transfer curves in the lowinput-voltage region, $\left|V_{\text {in }}\right|<0.5 \mathrm{~V}$, became sharp with decreasing $V_{\mathrm{G}}$. At $V_{\mathrm{G}}=-1 \mathrm{~V}$, the slope in this region was unity. On the other hand, the curves in the high-input-voltage region, $\left|V_{\text {in }}\right|>1$ $\mathrm{V}$, were almost the same as the curves for $V_{\mathrm{G}}>-0.6 \mathrm{~V}$. The slope in this region was again unity. The obtained results indicate that there were two mechanisms controlling voltage transfer characteristics and they were switched by changing the input voltage.

In order to examine the effect of WPGs in detail, $V_{\mathrm{G}}$ on each side was varied independently, keeping that on the other side at $+0.2 \mathrm{~V}$. Measured voltage transfer curves are plotted in Figs. 5(b) and 5(c) while changing the WPG voltage on left and right branches, respectively. Curves on only one side of the input voltage polarity were modulated by $V_{\mathrm{G}}$. The output on the other side was slightly positive in the low-input-voltage region. Considering the position of the WPG and the polarity of the input voltage, it was found that the WPG on the positively biased branch modulated outputs and that on the other branch was not effective. Interestingly, curves on modulated sides in Figs. 5(b) and 5(c) were quite similar to the curves in each side of the plot in Fig. 5(a). These results indicate that only the WPG on the positively biased branch controlled voltage transfer characteristics even in the case of two WPGs. ${ }^{11}$

The gate voltage dependence of the curvature from fitting with a parabolic function is shown in Fig. 6. The curvature increased rapidly when $V_{\mathrm{G}}<-0.6 \mathrm{~V}$, although a small change was seen when $V_{\mathrm{G}}>-0.6 \mathrm{~V}$. By comparing with branch current as shown in Fig. 6, a rapid change of the curvature was found to take place in the subthreshold region. In this region, a potential barrier was formed underneath the WPG and only thermally excited carriers could flow. The formation of the potential barrier by depletion seemed to give a critical change in voltage transfer characteristics. 


\section{Discussion}

\subsection{Mechanism and modeling}

On the basis of the ballistic transport model, it is difficult to explain the appearance of nonlinear characteristics in the devices larger than the mean free path and their RT operation. Therefore, in this study, an alternative model for the present devices is introduced. Its concept is shown in Fig. 7. Basically, the nonlinearity of the voltage transfer curve in TBJ devices comes from the asymmetry of resistance in two input branches. Assuming resistances under WPGs for the left and right branches, $r_{L}$ and $r_{\mathrm{R}}$, respectively, the output voltage is given by the simple formula,

$$
V_{\text {out }}=-\frac{r_{L}-r_{R}}{r_{L}+r_{R}} V_{i n} .
$$

In the case of the previous theory, the asymmetry of the resistance is given by the difference in the number of occupied quantum channels for different electrochemical potentials. ${ }^{1}$ In the present device, it is given by the asymmetry of potential inside due to two WPGs with equal gate voltage. Thus, the nonlinearity can be seen even in the diffusive regime. As shown in Fig. 7(a), potential hills are formed underneath WPGs and are almost fixed by $V_{\mathrm{G}}$. When input voltages are applied to right and left branches in push-pull fashion, the potential hill remains only in the positively biased branch. The hill in the negatively biased branch is cancelled by negative input voltage as shown in Fig. 7(b). Then, the resistance in the positively biased branch becomes higher than that in the negatively biased branch. By switching the polarity of the input, the high-resistance branch is also switched.

For quantitative discussion, the voltage transfer characteristic is formularized on the basis of the present model. Considering the potential diagram in Fig. 7(b), the density of carriers passing underneath the WPG in the negatively biased branch [right branch in Fig. 
7(b)] is evaluated using $n_{\mathrm{R}}=n_{0} \exp \left[\left(E_{\mathrm{FR}}-E_{\mathrm{F} 0}\right) / k T\right]$, where $n_{0}$ and $E_{\mathrm{F} 0}$ are the initial carrier density and Fermi energy without $V_{\mathrm{in}}$, respectively, and $E_{\mathrm{FR}}$ is the Fermi energy in the right branch. On the other hand, that in the positively biased branch is given by $n_{\mathrm{L}}=n_{0} \exp \left[\left(E_{\mathrm{F} 0^{-}}\right.\right.$ $\Delta E) / k T]$, where $\Delta E$ is the height of the potential hill underneath the WPG. Because the resistance is proportional to the inverse of the carrier density, $V_{\text {out }}$ can be evaluated using eq. (1). Taking account of the unintentional voltage drop due to series resistance and other factors, $V_{\text {out }}$ is given by the next formula:

$$
V_{\text {out }}=-V_{\text {in }} \tanh \left(-\frac{\beta V_{\text {in }}}{2 k T}\right)
$$

where $\beta$ is the input-voltage-to-energy scaling factor defined by $\Delta E / \Delta V_{\text {in }}$, and $0 \leq \beta \leq 1$. This equation is applicable to the nonballistic condition. $\beta$ is a unique parameter for describing the difference in characteristics. Typical curves at 100 and $300 \mathrm{~K}$ based on the present model are plotted in Fig. 7(c). For comparison, theoretical curves, $V_{\text {out }}=-\alpha V_{\text {in }}{ }^{2} / 2$, based on the ballistic transport model from ref. 1 are also plotted, which are applicable only in the low input voltage region due to the second-order approximation. A significant difference between the two models is seen in the temperature dependence. Namely, a clear temperature dependence is predicted in the present model, although it is not seen in the previous model. It can reproduce the experimental curves well even in the high-input-voltage region, where the output shows an almost linear curve and detailed analysis in the previous model also explains it. ${ }^{15}$

\subsection{Analysis of experimental results}

First, the experimental characteristics at various temperatures in Fig. 6 were compared with the present model. The experimental and theoretical curves are plotted for two devices with different sizes in Fig. 8(a). By simply choosing a suitable $\beta$, the present model could successfully reproduce the whole curves at various temperatures for each device. This result clarifies the validity of the present model. The difference in temperature dependence between 
two devices was understood to be due to the difference in $\beta$. To see the behavior in detail, the curvatures were evaluated individually by fitting the experimental data using the parabolic function and compared with the model. Evaluated $\alpha$ as a function of temperature is plotted in Fig. 8(b). It was found that $\alpha$ increased with decreasing temperature. The temperature dependence of $\alpha$ can be obtained by taking the Taylor expansion of eq. (2) and comparing it with the coefficient of the second-order term of the expanded formula,

$$
\alpha=\beta / k T \text {. }
$$

This equation indicates that the curvature increases when temperature decreases. In the case of the device with $W=570 \mathrm{~nm}$, the theoretical curve depicted by a solid line can explain $\alpha$ in the whole measured temperature range with a constant $\beta$ of 0.022 . In the case of the device with $W=120 \mathrm{~nm}$, evaluated $\alpha$ followed the theory with $\beta=0.06$, when $T>100 \mathrm{~K}$. However, when $T<100 \mathrm{~K}, \alpha$ became smaller than the theory and showed a rather small temperature dependence. The mean free path at $77 \mathrm{~K}$ was $1,500 \mathrm{~nm}$, which was 10 times larger than the device size, and the transport was ballistic. The ballistic model ${ }^{1}$ showed a smaller temperature dependence than the present model. Thus, the nonlinear mechanism was considered to change from the present model to the ballistic one. On the basis of a comparison of the nanowire width and the mean free path, switching of the dominant mechanism is expected to take place between 200 and $300 \mathrm{~K}$ in the narrow device. This seemed to reflect the slight jump of curves from 300 to $200 \mathrm{~K}$ in Fig. 4(a), although the change of $\alpha$ was gradual. Detailed evaluation of the temperature dependence of the mobility will make more quantitative discussion possible.

Next, the size dependence of the voltage transfer curves is discussed on the basis of the present model. Again, the model could reproduce the experimental curves in Fig. 2(a) well when we choose suitable $\beta$ values. Evaluated $\beta$ obtained by fitting showed a clear correlation with $\alpha$ in Fig. 3. Taking a plot of $\beta$ vs $\alpha$, we found $\beta / \alpha$ to be $0.0255 \mathrm{eV}$ at RT, which could be accurately explained by eq. (3). Then, the $W$ dependence of voltage transfer curves is 
understood by the change of the $W$ dependence of $\beta$. A possible mechanism relating $W$ and $\beta$ is the $W$ dependence of the potential hill underneath the WPG, $\Delta E$. In the present model, the nonlinearity comes from the existence of $\Delta E$ even though it does not appear in eq. (2). When $\Delta E$ becomes small, the asymmetry of the potential disappears and the curvature and $\beta$ decreases. This relationship can be verified by the analysis of the equivalent circuit of the present device consisting of a couple of FETs connected in series in the linear region,

$$
V_{\text {out }} \approx\left(V_{G}-V_{\text {th }}\right)-\sqrt{\left(V_{G}-V_{\text {th }}\right)^{2}+V_{\text {in }}^{2}}
$$

where $V_{\text {th }}$ is the threshold voltage of the two WPGs. It was found that $\beta$ is given by $k T /\left(V_{\mathrm{G}^{-}}\right.$ $V_{\text {th }}$ ) from the expansion of eq. (4). It is obvious that decreased $V_{\text {th }}$ corresponds to small $\Delta E$. Therefore, small $\Delta E$ results in small $\beta$. On the other hand, it is known that the threshold voltage of WPG decreases when $W$ increases. ${ }^{16}$ This means that $\Delta E$ is small in the device having large $W$. Then, $\beta$ is found to decrease with increasing $W$. The discussion above can qualitatively explain the $W$ dependence of $\beta$, although further discussion is necessary for a quantitative analysis of the size dependence of the nonlinear characteristics.

The gate voltage dependence of the voltage transfer curves is also analyzed, focusing on the appearance of the complex curve. $\beta$ as a function of $V_{\mathrm{G}}$ in the low-input-voltage region was evaluated by fitting the experimental curves with eq. (2) and it showed a curve following $\beta=\alpha k T$. On the other hand, $\beta$ in the high-voltage region was almost constant. From the comparison with the branch current, the condition of the WPG was in the subthreshold region when $V_{\mathrm{G}}<-0.6 \mathrm{~V}$. A possible band diagram in the subthreshold region is shown in Fig. 9(a). If $V_{\mathrm{G}}$ is less than $V_{\text {th }}$, the WPG completely depletes the channel and conductance goes to zero at $V_{\text {in }}=0 \mathrm{~V}$, resulting in a capacitive connection rather than a resistive one. However, in the negatively biased branch, the input voltage cancels the gate voltage and the potential barrier disappears, resulting in a resistive connection. Then, the device can be treated as an equivalent circuit shown in Fig. 9(b). In this case, $V_{\text {out }}=-V_{\text {in }}$, that is, $\Delta V_{\text {out }} / \Delta V_{\text {in }}=1$, is realized because 
the capacitance in the positively biased branch acts as a high resistance. Increasing $V_{\text {in }}$, the positively biased branch eventually switches to a resistive one again by barrier lowering or tunneling due to high-positive-voltage application. The equivalent circuit goes back to that in Fig. 9(c) and the curve is again given by eq. (2). Then, the voltage transfer curve in the subthreshold region has the feature of two different curves as shown in Fig. 5(a). Additionally, the slight positive $V_{\text {out }}$ and lateral shift of the peaks in the case of asymmetric WPG voltage application in Figs. 5(b) and 5(c) can be explained by the asymmetry of $\Delta E$ in the left and right branches in the present model, determined using eq. (1) and carrier density. The asymmetry of the voltage transfer curves in Figs. 2(a) and 4 can be attributed to the fluctuation of the wire width and/or the threshold voltage shift.

\section{Conclusions}

The nonlinear electrical characteristics of GaAs-based three-branch nanowire junction (TBJ) devices having Schottky wrap gates (WPGs) were investigated experimentally and theoretically, focusing on the nonlinear mechanism at room temperature for devices with large dimensions and the improvement of voltage transfer efficiency. Nanowire width, temperature, and WPG gate voltage dependences of input-output voltage transfer curves, $V_{\text {out }}-V_{\text {in }}$, were characterized systematically. Bell-shaped $V_{\text {out }}-V_{\text {in }}$ voltage curves were observed at room temperature even in devices having a nanowire width of 1,500 $\mathrm{nm}$. With decreasing wire width or temperature, the output curves were sharpened and the curvature from fitting based on a parabolic function increased. With decreasing WPG gate voltage, $V_{\mathrm{G}}, V_{\text {out }}-V_{\text {in }}$ curves in the low-input-voltage region also became sharp. The curvature rapidly increased and voltage transfer efficiency, $\Delta V_{\text {out }} / \Delta V_{\text {in }}$, reached unity when $V_{\mathrm{G}}$ decreased in the subthreshold region. A simple and compact model for the nonlinear characteristics in the nonballistic regime was 
introduced and it showed that the difference in the voltage transfer characteristics was translated into only a unique parameter, $\beta$. The rapid change of the curvature and complex curve in the subthreshold region under $V_{\mathrm{G}}$ control was found to be due to the switching of the branch condition from resistive to capacitive owing to depletion underneath the WPG.

\section{Acknowledgments}

The authors thank Professor T. Fukui and Professor T. Hashizume for their continuous support. This work was partly supported by a Grant-in-Aid for Young Scientists (A) (17686028) from the Ministry of Education, Culture, Sports, Science and Technology, Japan. 


\section{References}

1) H.-Q. Xu: Appl. Phys. Lett. 78 (2001) 2064.

2) J.-O. J. Wesström: Phys. Rev. Lett. 82 (1999) 2564.

3) I. Shorubalko, H.-Q. Xu, I. Maximov, P. Omiling, L. Samuelson, and W. Seifert: Appl. Phys. Lett. 79 (2001) 1384.

4) L. Worschech, A. Schliemann, S. Reitzenstein, P. Hartmann, and A. Forchel: Microelectron. Eng. 63 (2002) 217.

5) C. Papadopoulos, A. Rakitin, J. Li, A. S. Vedeneev, and J. M. Xu: Phys. Rev. Lett. 85 (2000) 3476.

6) L. Bednarz, Rashim, I. Hynene, T. Gonzalez, and J. Mateos: IEEE Trans. Nanotechnol. 5 (2006) 750 .

7) H. Q. Xu, I. Shorubalko, D. Wallin, I. Maximov, P. Omiling, L. Samuelson, and W. Seifert: IEEE Electron Device Lett. 25 (2004) 164.

8) S. Reitzenstein, L. Worschech, and A. Forchel: IEEE Electron Device Lett. 24 (2003) 625.

9) I. Shorubalko, H. Q. Xu, I. Maximov. D. Nilsson, P. Omling, L. Samuelson, and W. Seifert: IEEE Electron Device Lett. 23 (2002) 377.

10) J. Sun, D. Wallin, P. Brusheim, I. Maximov, Z.-G. Wang, and H.-Q. Xu: Nanotechnology 18 (2007) 195205.

11) T. Nakamura, Y. Shiratori, S. Kasai, and T. Hashizume: Appl. Phys. Lett. 90 (2007) 102104.

12) D. Wallin, I. Shorubalko, H. Q. Xu, and A. Cappy: Appl. Phys. Lett. 89 (2006) 092124.

13) J. Mateos, B. G. Vasallo, D. Pardo, T. Gonzalez, J.-S. Galloo, S. Bollaert, Y. Roelens, and A. Cappy: IEEE Trans. Electron Devices 50 (2003) 1897.

14) S. Kasai, K. Jinushi, H. Tomozawa, and H. Hasegawa: Jpn. J. Appl. Phys. 36 (1997) 1678.

15) H. Q. Xu: Physica E 13 (2002) 942. 
16) Y. Shiratori and S. Kasai: presented at the 2007 Int. Conf. Solid State Devices and Materials, Tukuba, Sep. 19-21, 2007; to be published in Jpn. J. Appl. Phys. 47 (2008). 


\section{Figure captions}

Fig. 1. (a) Basic structure of a TBJ device having Schottky wrap gates (WPGs), (b) WPG structure, and (c) SEM image of a fabricated device.

Fig. 2. (a) Voltage transfer curves for various nanowire widths and (b) their derivatives.

Fig. 3. Curvature as a function of nanowire width, $W$.

Fig. 4. Voltage transfer curves for various temperatures in TBJ devices with (a) $W=120 \mathrm{~nm}$ and (b) $W=570 \mathrm{~nm}$.

Fig. 5. Voltage transfer curves for (a) common gate voltage application, and [(b) and (c)] for independent gate voltage application on left and right WPGs, respectively.

Fig. 6. Gate voltage dependence of curvature of outputs and branch current.

Fig. 7. A model for the nonlinear voltage transfer curve in the TBJ device controlled by WPGs. Potential diagrams at (a) $V_{\text {in }}=0 \mathrm{~V}$ and (b) $V_{\text {in }}>0 \mathrm{~V}$, and (c) example of calculated curves.

Fig. 8. (a) Experimental and theoretical voltage transfer curves for the devices with $W=120$ and $570 \mathrm{~nm}$, and (b) evaluated $\alpha$ and $\beta$ as a function of temperature.

Fig. 9. (a) Potential diagram in the subthreshold region, and equivalent circuits (b) in the lowinput-voltage region and (b) in the high-input-voltage region. 


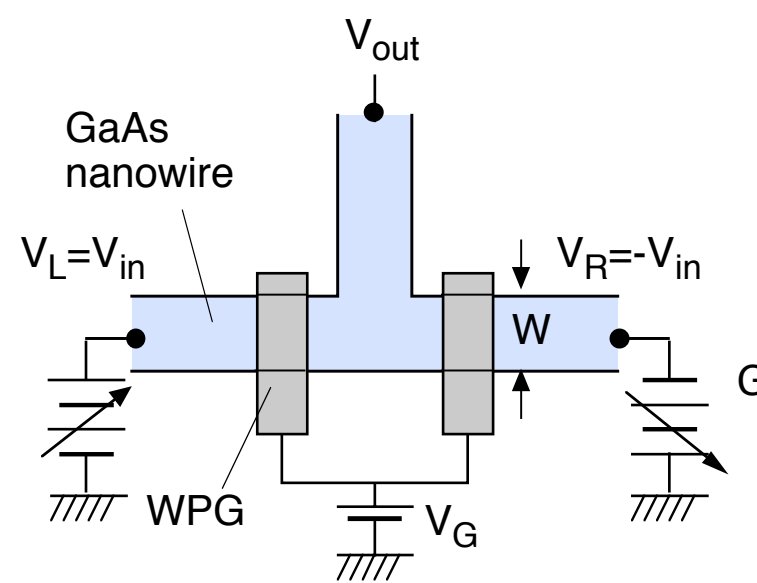

(a)

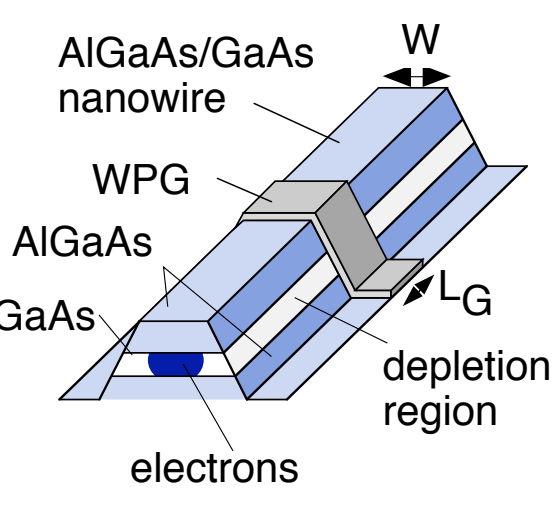

(b)

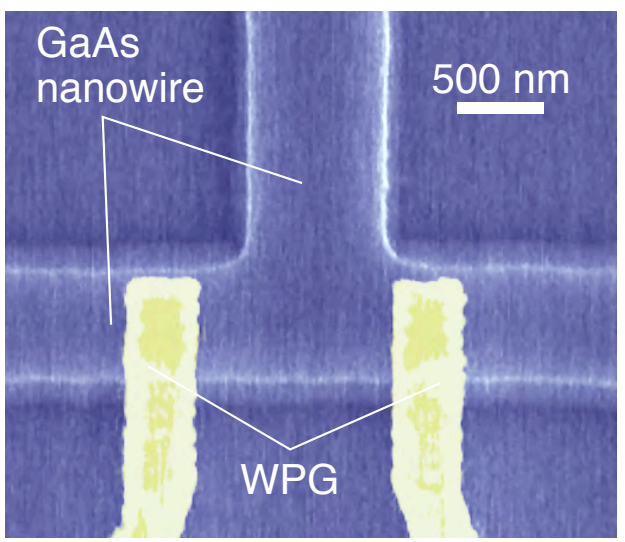

(c)

Kasai et al., Figure 1 


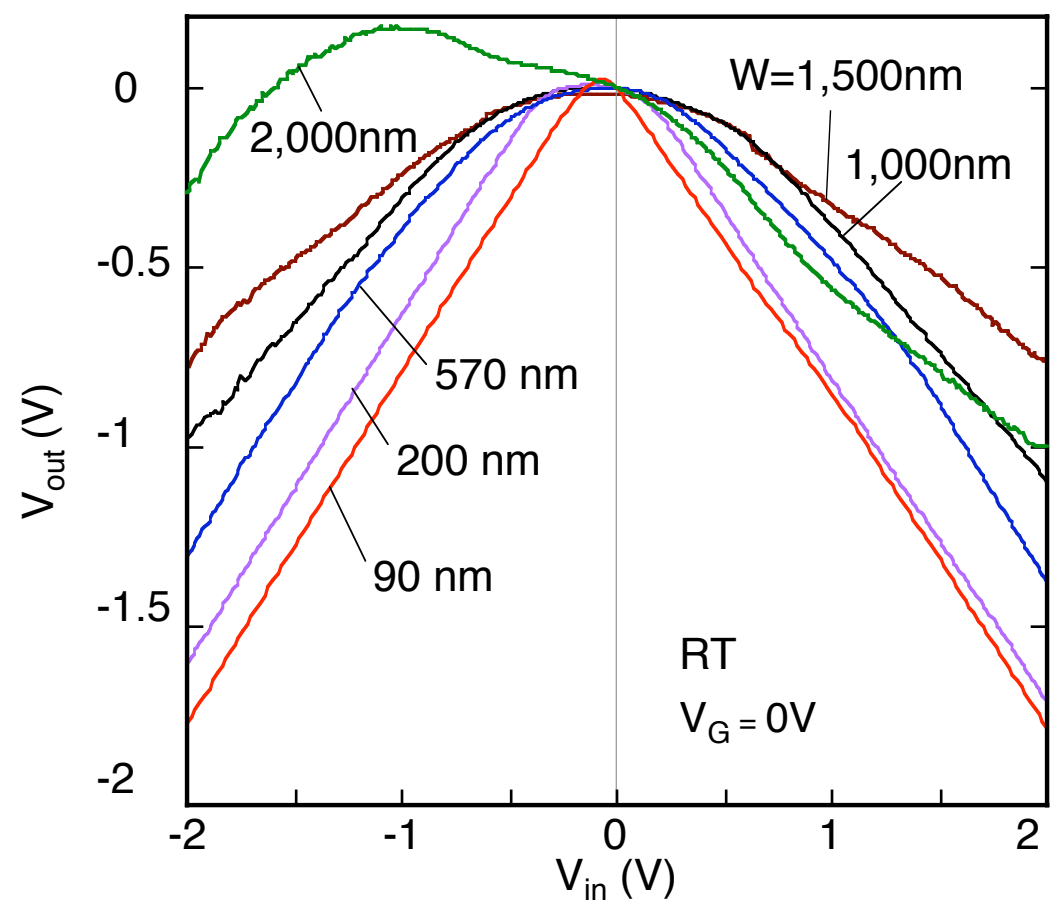

(a)

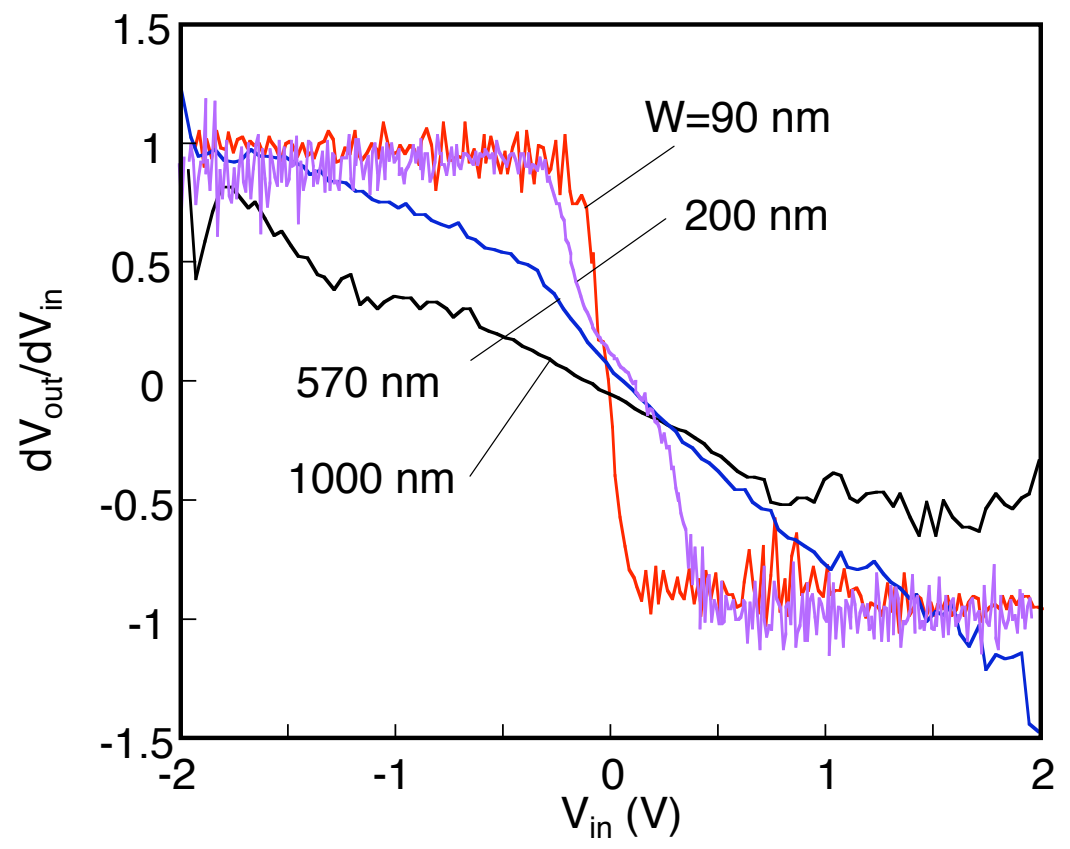

(b)

Kasai et al., Figure 2 


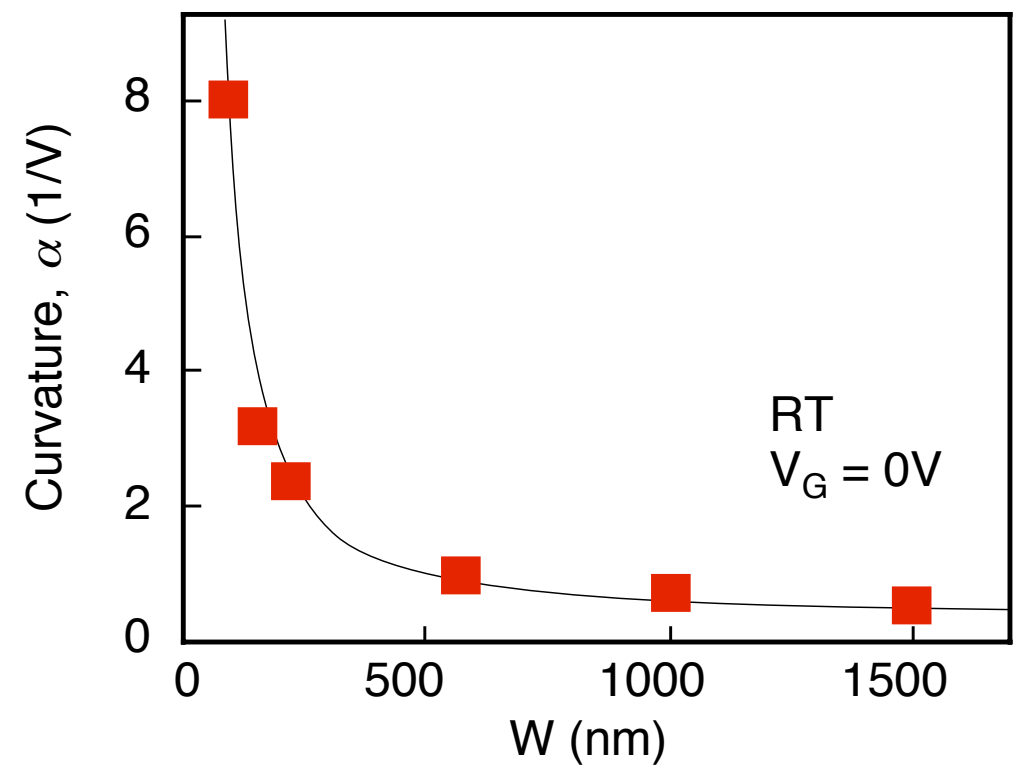

Kasai et al., Figure 3 


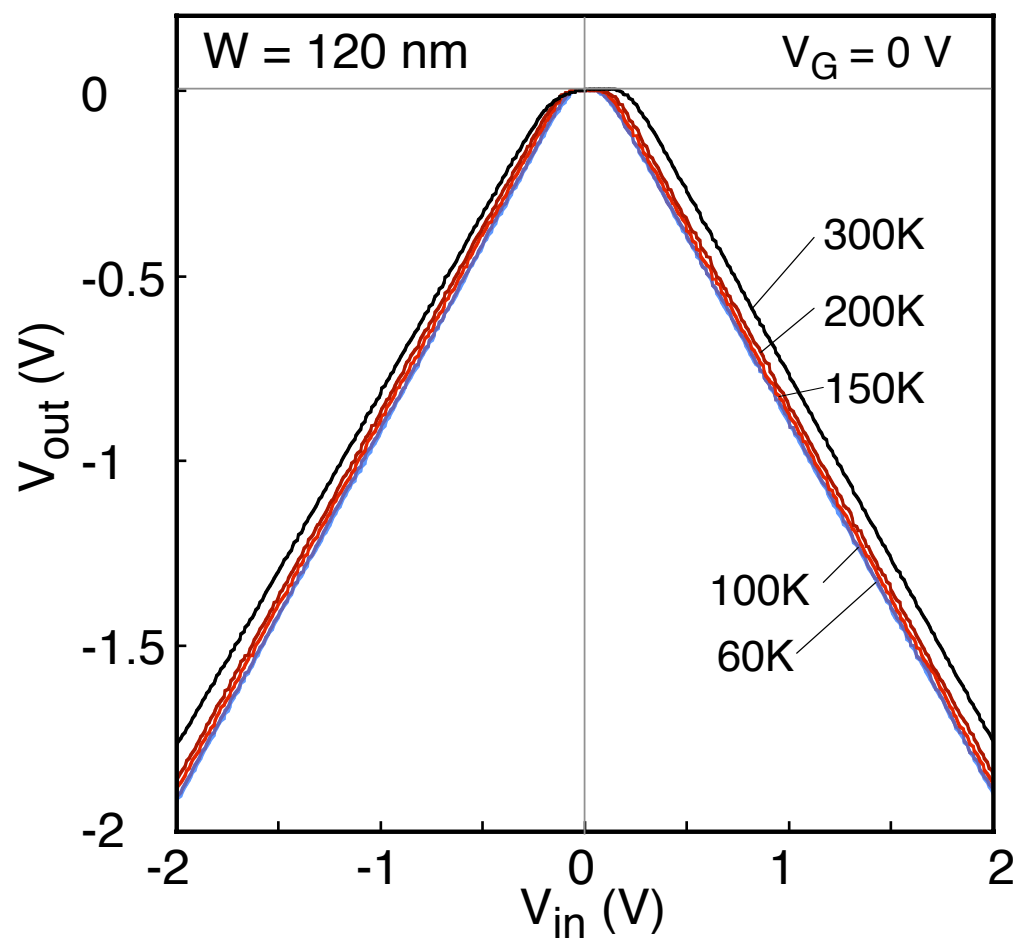

(a)

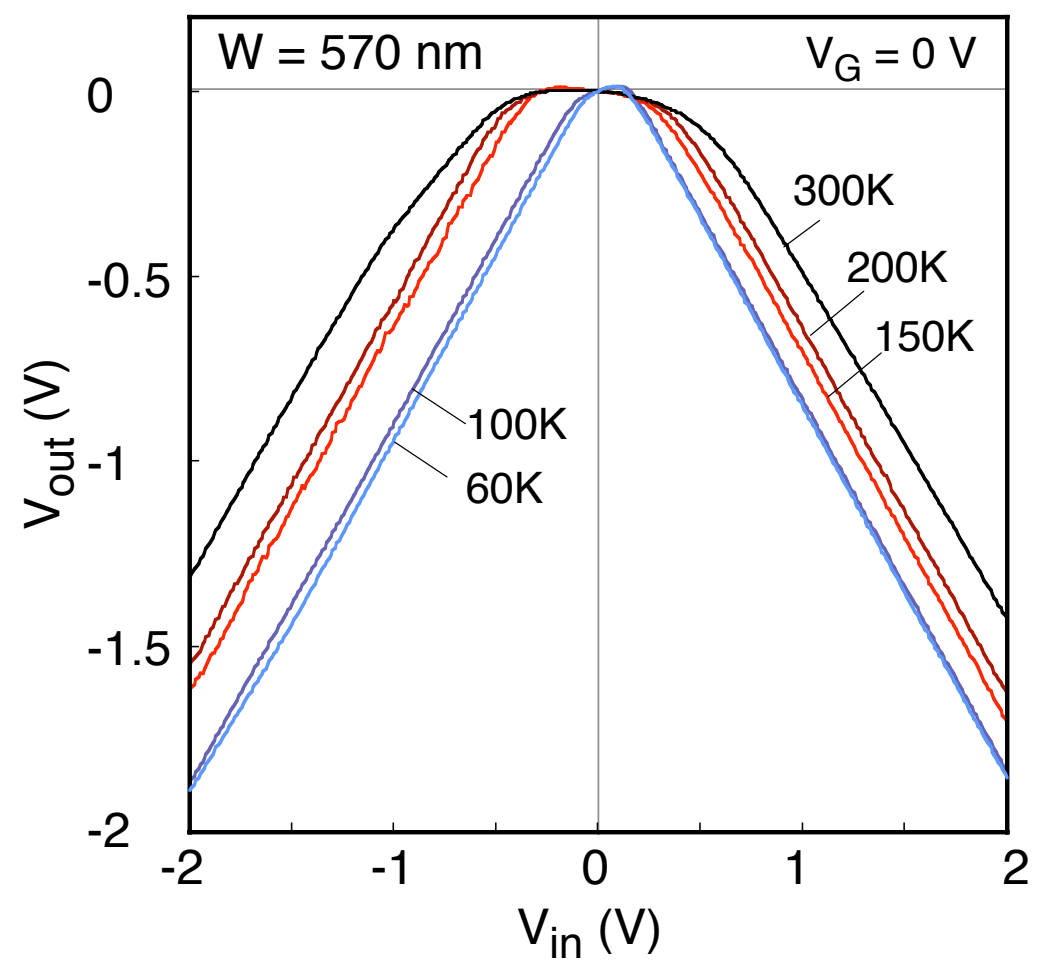

(b)

Kasai et al., Figure 4 


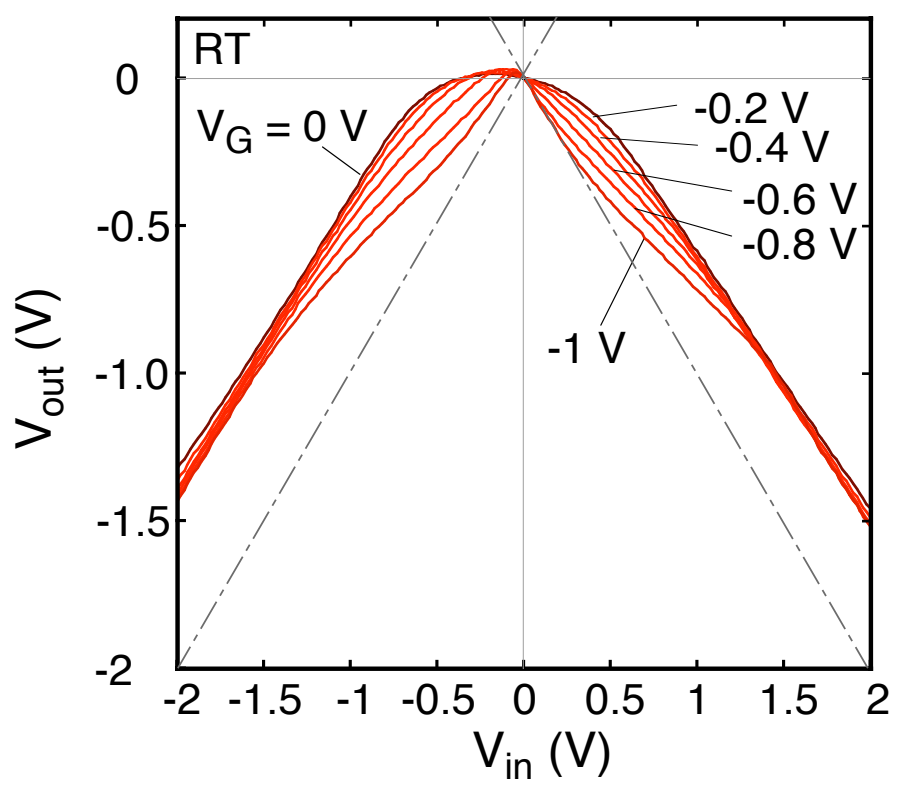

(a)

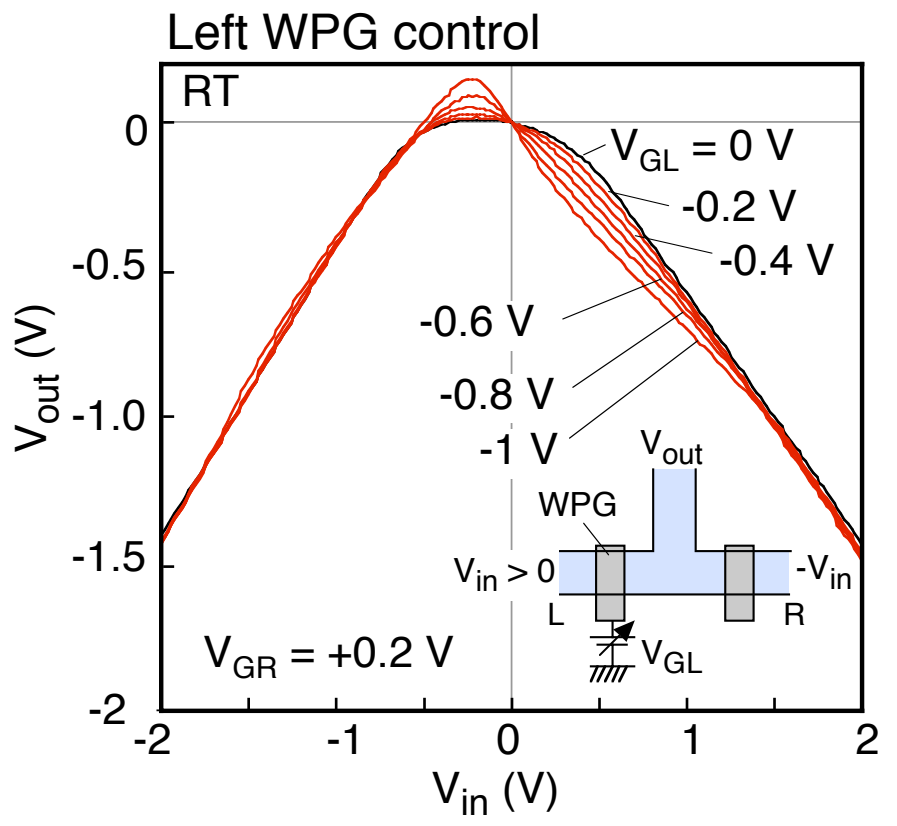

(b)

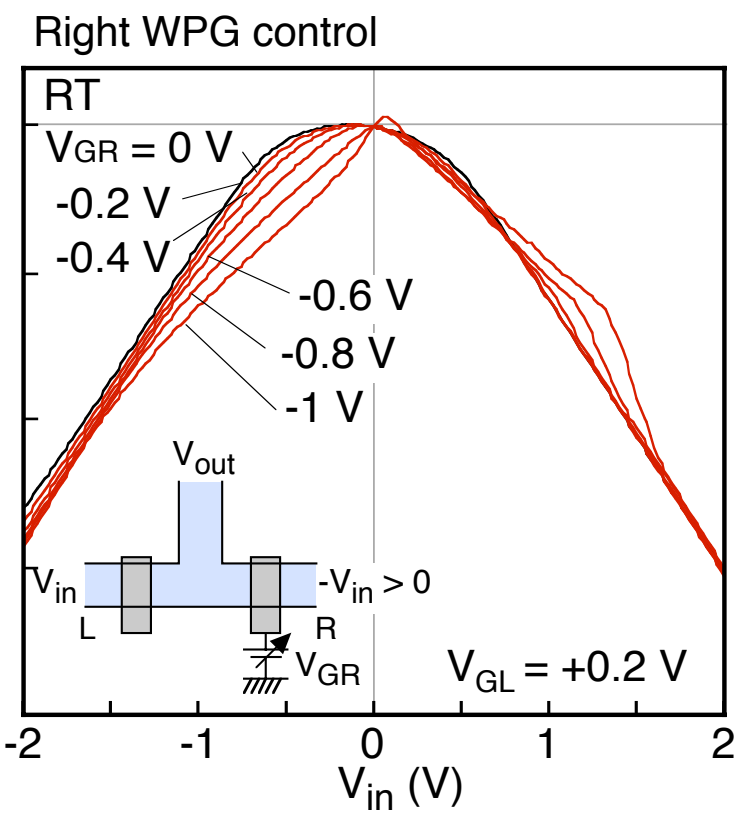

(c)

Kasai et al., Figure 5 


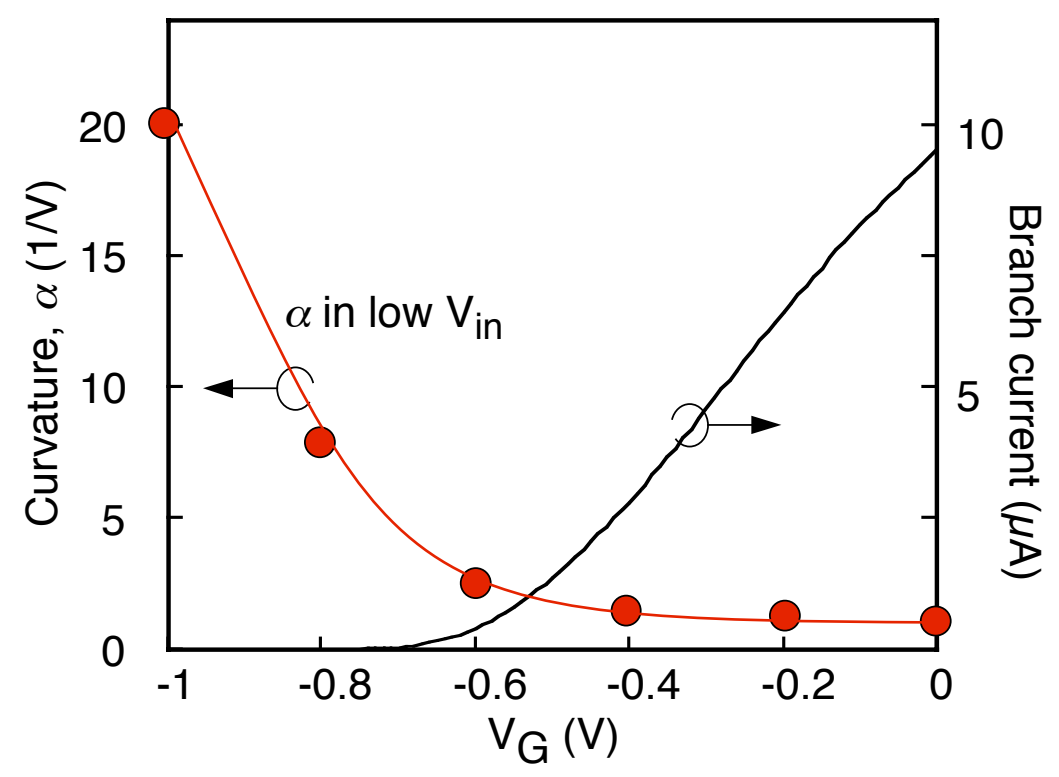

Kasai et al., Figure 6 


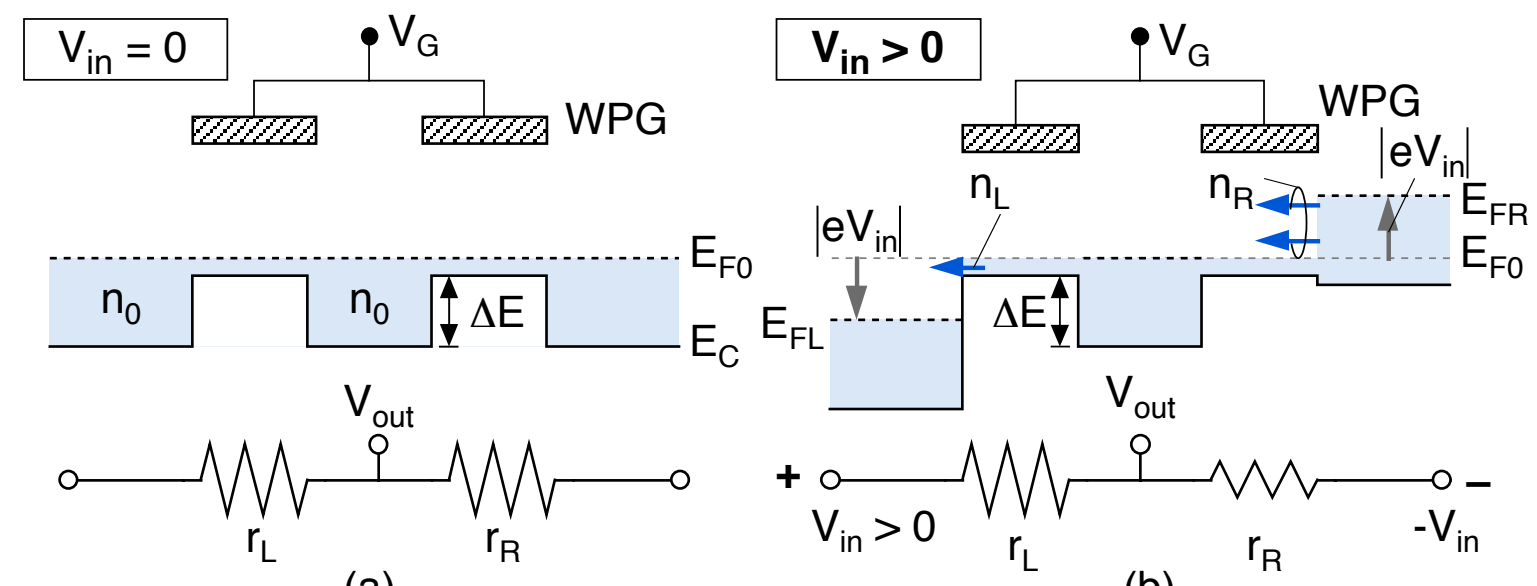

(a)

(b)

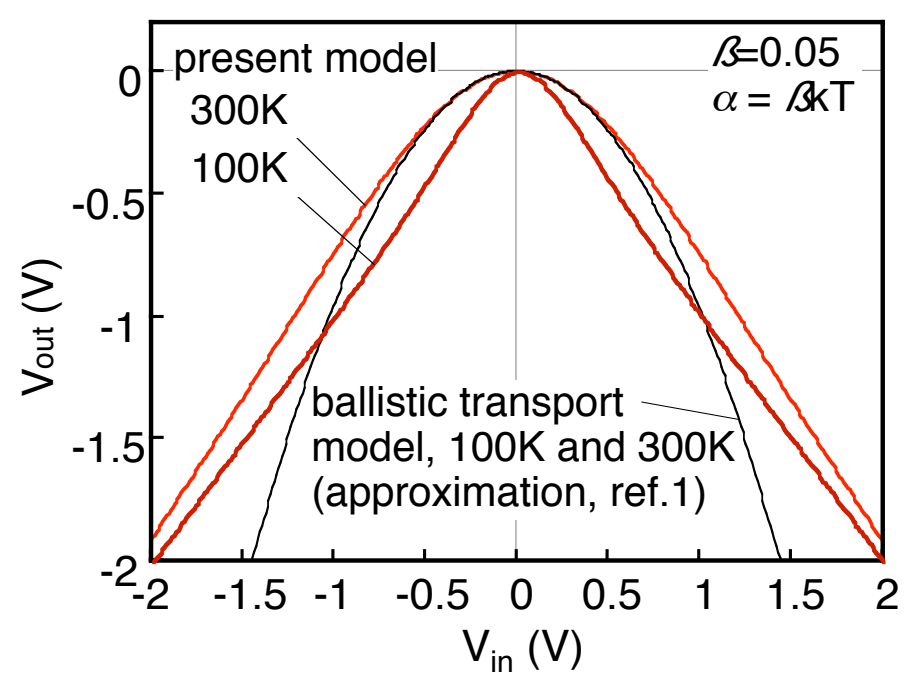

(c)

Kasai et al., Figure 7 

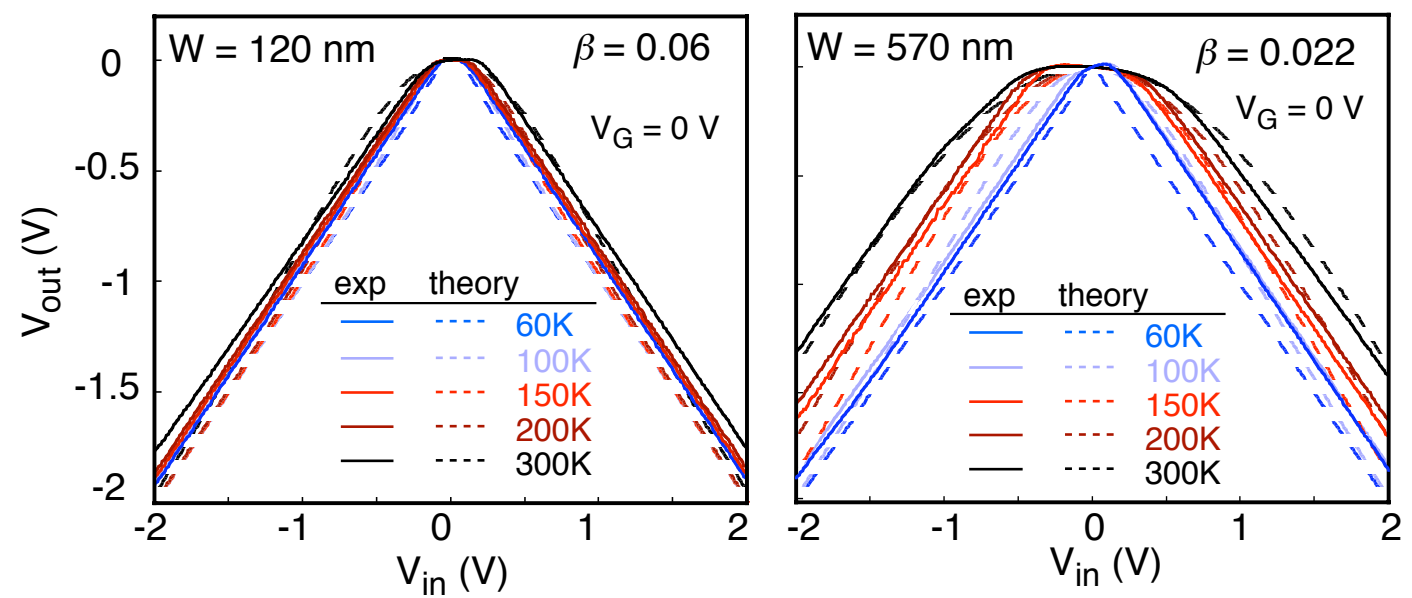

(a)

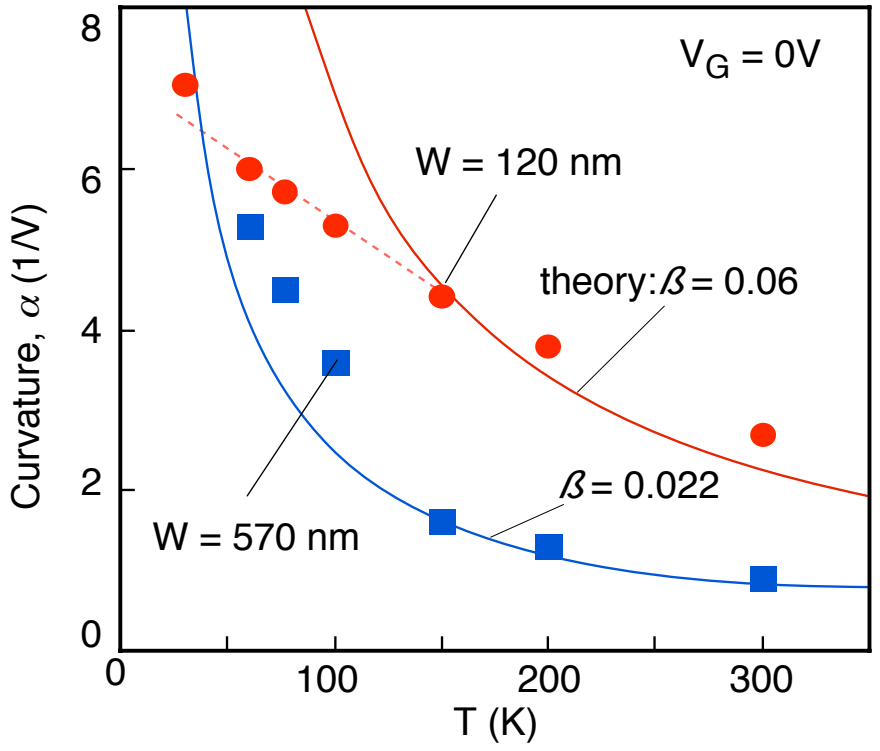

(b)

Kasai et al., Figure 8 


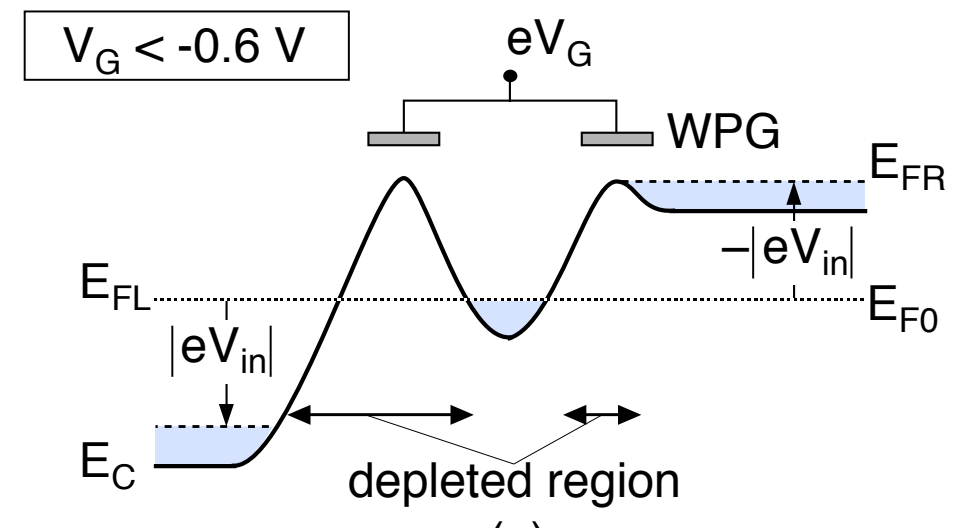

(a)

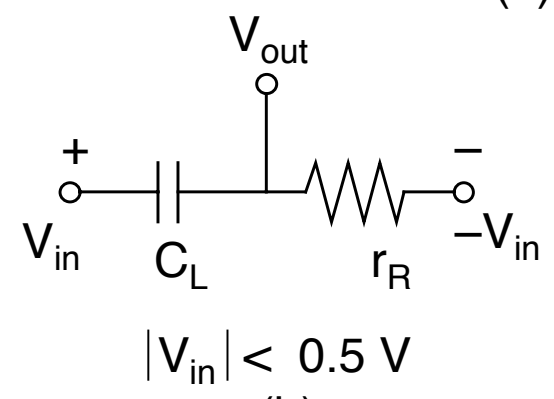

(b)

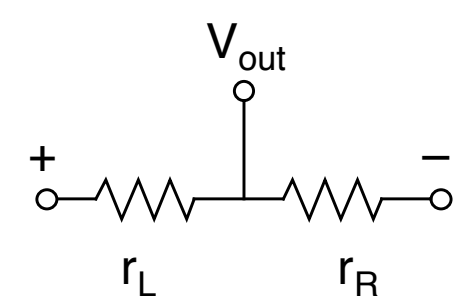

$\left|\mathrm{V}_{\text {in }}\right|>1.0 \mathrm{~V}$

(c)

Kasai et al., Figure 9 